\title{
Comparison of Th17 cells mediated immunological response among asthmatic children with or without allergic rhinitis
}

\author{
Miao Qing, ${ }^{1}$ Liu Yongge, ${ }^{1} \mathrm{Xu}$ Wei, ${ }^{1}$ WANG Yan,${ }^{1}$ Li Zhen,${ }^{1}$ Ren Yixin,,${ }^{1}$ Guan Hui,${ }^{1}$ Xiang ${ }^{1}$
}

\begin{abstract}
Objective: To investigate whether there were differences in Th17 cells mediated immunological responses among asthmatics with or without allergic rhinitis.

Methods: A case-control comparison was conducted in a cohort of 67 children with asthma (AS), 50 children with allergic rhinitis (AR), 52 children with both AS and AR (ASR), 25 infectious rhinitis (IR), and 55 healthy controls (HC). The percentages of circulating Th17 cells were determined by flow cytometry. The Th2- and Th17-related cytokines in plasma and culture supernatants were measured by enzyme-linked immunosorbent assay. The effect of proinflammation cytokine IL-17E on Th2 cytokines production from human T helper (Th) lymphocytes was analyzed.
\end{abstract}

Results: (1) A inter-group comparison revealed that Th17 cells levels were highest in ASR group [(0.89\% \pm 0.27$) \%]$, following by AS group $[(0.82 \pm 0.29) \%]$ and AR group [ $(0.78 \pm 0.17) \%](P<0.05)$. (2) After in-vitro stimulation with house dust mite (HDM) antigen, the levels of IL-4 and IL-17E in culture supernatants of PBMCs from allergic children (AS group, AR group and ASR group) were significantly enhanced. (3) The release of Th2 cytokines from IL-17E treated Th cells of allergic children (AS group, AR group and ASR group) were significantly induced, no similar result was observed in IR group and HC group.

Conclusion: Our findings preliminarily revealed that Th17 cell and its related cytokines might be involved in pathogenesis of airway inflammation diseases, and also presenting varying immunological characteristics among asthmatic children with or without allergic rhinitis.

Keywords: allergic rhinitis, bronchial asthma, infectious rhinitis, Th17 cells, interlukin-17A, interlukin-17E, interlukin-17F

From:

Allergy Department, Beijing Children's hospital, Capital Medical

University, Beijing, China, No.56 Nanlishi Road, Xicheng District, 100045, Beijing P.R.China

\section{Background}

Airway allergic diseases, including allergic rhinitis (AR) and asthma (AS), constitute a heterogeneous group of chronic inflammatory disorders, which are driven by various immune cells and inflammatory mediators. ${ }^{1-2}$ In clinical practice, it is common that both AS and AR frequently occurs together in one individual. Several studies have reported that the prevalence of $\mathrm{AR}$ in asthmatic children ranges from $30 \%$ to $70 \% .^{3-4}$ Therefore, the Allergic Rhinitis and Its Impact on Asthma Workshop Group published an international consensus statement ([ARIA] guideline) in 2001, proposing that AR and AS should be considered as the manifestations of One Airway, One Disease and applied an integrated diagnosis and therapeutics protocol..$^{5-6}$

\author{
Corresponding author: \\ Xiang Li \\ Allergy Department \\ Beijing Children's Hospital, Capital Medical University \\ No.56 Nanlishi Road, Xicheng District,100045, Beijing P.R.China \\ E-mail: drxiangli@163.com
}

The "Th1/Th2" imbalance has been widely considered to be central to the pathogenesis of airway allergic diseases, pointing to an improper immunological response mediated by Th2 cells in local microenvironment. ${ }^{7-8}$ Recently, a unique subset of $\mathrm{CD}^{+} \mathrm{IL}-17 \mathrm{~A}^{+} \mathrm{T}$ cells, termed Th17 cells, has recently drawn attention as possible effector cell in inflammatory process. ${ }^{9-10}$ A number of studies supported that either an inappropriate function Th17 cells or increased levels of Th17-related cytokines were involved in pathologic mechanism among children with AS or AR alone. ${ }^{11-12}$ However, few studies have explored whether there were differences existing in Th17 cells immunity among children with both AS and AR. Therefore, in present 
study, we aimed to compare the numbers of Th17 cells and the concentrations of Th17-related cytokines (IL-17A, IL-17E and IL-17F) among children with AS alone(AS), with AR alone(AR), or those with both AS and AR together (ASR).

\section{Methods and Materials Subjects}

A total of 169 children suffering from allergic airway diseases (67AS, 50AR, and 52 ASR) who had been sensitized to house dust mites (HDM) were chosen from the Allergic Outpatient Clinic at Beijing Children's Hospital between January and June 2015. The diagnosis and assessment of AS was evaluated according to the Global Initiative for Asthma (GINA) criteria. ${ }^{13}$ AR was diagnosed according to the ARIA guideline. ${ }^{14}$ The diagnosis of ASR was performed in the followings steps: children with doctor-diagnosed AS were transferred to the Otolaryngology Head and Neck Surgery Outpatient Clinic, where a positive diagnosis based on their upper airway symptoms was provided by the rhinologists. Sensitization to aeroallergens were identified by the skin prick test(SPT); a positive result of SPT was defined as a wheal greater than or equal to half of the diameter of the positive control (histamine, $10 \mathrm{mg} / \mathrm{mL}$ ) and at least $3 \mathrm{~mm}$ larger than the diameter of the negative control (saline). Another 25 children suffering from infectious rhinitis (IR) with nasal symptoms of respiratory tract infection, including nasal congestion, runny nose, and snoring, were chosen from the ENT Outpatient Clinic at Beijing Children's Hospital. All IR patients had negative SPT results and undetectable sIgE.

55 age- and sex-matched healthy children were enrolled as the healthy control (HC) group, who had normal spirometry tests and negative SPT results, no history of allergic symptoms, and no history of upper or lower airway disease. Exclusion criteria were (1) an acute infection within the last 4 weeks; (2) treatment with oral or intravenous steroids within last 3 months, or (3) a history of immunotherapy for any allergens within the past 3 years.

All enrolled subjects were fully informed of the purpose and nature of the study, which were approved by the medical ethnic committee of Beijing Children's Hospital. Written informed consent was obtained from all parents/guardians of all the participants.

\section{Isolation of Peripheral Blood Mononuclear Cells (PBMCs) and $\mathrm{CD}^{+}$Th cells}

Five milliliters of human peripheral venous blood, obtained from each enrolled child, was diluted 1:2 with phosphate -buffered saline (PBS), then was centrifuged using Ficoll density gradient solution (Haoyang Biotech, Tianjin,China) at $2000 \mathrm{rpm}$ for 20 minutes at $4^{\circ} \mathrm{C}$ to isolate the PBMCs. The PBMCs were collected and washed twice with cold PBS containing 2\% fetal calf serum (Gibco BRL Co. Ltd., USA); they were then resuspended in RPMI 1640 medium (Invitrogen Corp., CA, USA) supplemented with $100 \mathrm{U} / \mathrm{mL}$ penicillin (KeyGen Biotech, Nanjing, China) and $100 \mathrm{U} / \mathrm{mL}$ streptomycin (KeyGen), 2 mM glutamine (KeyGen), $20 \mathrm{U} / \mathrm{mL}$ rIL-2 (Novartis, Camberely, UK), and 10\% fetal calf serum (Gibco). Plasma samples were saved and stored at $-20^{\circ} \mathrm{C}$ for further cytokine measurements.

The untouched T-helper (Th) cells were negatively purified from PBMCs using a CD4 ${ }^{+}$T-cell isolation kit II (Miltenyi Biotec, Bergisch Gladbach, Germany) by passing them through a LS+ column (Miltenyi Biotec) in a magnetic field. In the resulting preparation the drop-through fraction contained $\mathrm{CD}^{+}$Th cells of a purity of at least $99 \%$ as assessed by flow cytometry.

\section{Cell culture}

After being cultured in 96-well round-bottomed plates in a concentration of $1.5 \times 10^{6} / \mathrm{mL}$, the PBMCs were incubated with $20 \mu \mathrm{g} / \mathrm{mL}$ Der p1 (Indoor Biotechnologies, Cardiff, UK), an allergen of HDM, in a final volume of $200 \mu \mathrm{L}$ per well for 96 hours at $37^{\circ} \mathrm{C}$ in a $5 \% \mathrm{CO}_{2}$ humidified incubator. Cells were harvested for intracellular cytokine staining, and the culture supernant before and after stimulation were collected and preserved at $-20^{\circ} \mathrm{C}$ for ELISA.

The isolated total Th cells were cultured with or without IL-17E $(50 \mathrm{ng} / \mathrm{mL})$ in the presence of costimulation $(10 \mu \mathrm{g} / \mathrm{mL}$ anti-CD3 antibodies and $1 \mu \mathrm{g} / \mathrm{mL}$ anti-CD28 antibodies) for 96 hours. The culture supernant were collected and preserved at $-20^{\circ} \mathrm{C}$ for ELISA.

\section{Intracellular Cytokine Staining of IL-17A}

The PBMCs were activated with $50 \mathrm{ng} / \mathrm{mL}$ phorbol-12 -myristate-13-acetate (PMA) (Sigma Chemical Co., St. Louis, MO, USA) and $1 \mu \mathrm{g} / \mathrm{mL}$ ionomycin (Sigma) for 96 hours at $37^{\circ} \mathrm{C}$ in a $5 \% \mathrm{CO}_{2}$ atmosphere; they were then stimulated for an additional 4 hours in the presence of $4 \mu \mathrm{M}$ monensin (Sigma). The cells were harvested, washed, and stained with APC-labeled anti-human CD4 (ebioscience, San Diego, CA) for 30 minutes on ice in the dark. After surface staining, the cells were fixed with fixation solution (ebioscience, San Diego, CA) and incubated for 20 minutes at room temperature in the dark with PE-labeled antihuman IL-17A antibody (ebioscience). PE-labeled rat IgG2a (ebioscience) was used as isotype control so as to enable correct compensation and confirm antibody specificity. Data were acquired on a FACS Calibur (BD Bioscience, San Diego, CA) and analyzed using FlowJo Software (FlowJo, Ashland, OR). The number of Th17 cells was expressed as a percentage of all the lymphocytes.

\section{Detection of Cytokines by ELISA}

The levels of Th2 cell-related cytokines (IL-4, IL-5 and IL-13) and Th17 cell-related cytokines (IL-17A, IL-17E, and IL-17F) in plasma or in cell-free supernatants were measured using ELISA kits as recommended by the manufacturer (ebioscience). All samples were measured in duplicate.

\section{Statistical Analysis}

Statistical analysis was performed using SPSS version 19.0 statistical software (IBM, Armonk, NY) and graphs were generated using prism software (GraphPad, La Jolla, CA). Qualitative variables were compared between the groups by chi-square tests and continuous variables were analyzed by the nonparametric Mann-Whitney $U$ test for unpaired comparisons. $P$ values of less than 0.05 were considered significant. 


\section{Results}

\section{Clinical Characteristics of Enrolled Subjects}

A total of 169 patients with airway allergic diseases (including: $67 \mathrm{AS}$ patients, $50 \mathrm{AR}$ patients and $52 \mathrm{ASR}$ patients), 25 children suffering from infectious rhinitis (IR) and another 55 healthy children were enrolled in this cross -sectional study. The clinical characteristics of the participants were shown in Table 1. No significant differences in age range, sex distribution, or BMI were found between patients groups (AS group, AR group, ASR group, and IR group) and HC group. However, the difference in $\mathrm{FEV}_{1} / \mathrm{FVC}$ ratio among these 5 groups were significant $(P<0.05)$.

\section{Percentages of circulating Th17 cells in PBMCs}

The frequency of blood Th17 cells $\left(\mathrm{CD} 4^{+} \mathrm{IL}-17 \mathrm{~A}^{+} \mathrm{T}\right)$ in children with allergic diseases, with infectious rhinitis and healthy controls were compared by flow cytometry (Figure 1). According to our results, the mean percentage of blood Th17 cells in PBMCs was significantly increased in 4 patient groups $[(0.75 \pm 0.23) \%]$ than that in HC group [ $0.2 \pm 0.1) \%](P<$ 0.05 , Figure 2). Moreover, through a inter-group comparison among patients groups, it revealed that the Th17 cells levels were highest in ASR group [ $(0.89 \% \pm 0.27) \%]$, following by AS group [(0.82 \pm 0.29$) \%]$ and AR group[(0.78 \pm 0.17$) \%]$, all of

Table1. The Participants' Clinical Characteristics

\begin{tabular}{lccccc}
\hline & ASR $(\boldsymbol{n}=\mathbf{5 2})$ & AS $(\boldsymbol{n}=\mathbf{6 7})$ & AR $(\boldsymbol{n}=\mathbf{5 0})$ & IR $(\boldsymbol{n}=\mathbf{2 5})$ & HC $(\boldsymbol{n}=\mathbf{5 5})$ \\
\hline Sex (boy/girl) & $31 / 21$ & $38 / 29$ & $17 / 34$ & $10 / 15$ & $28 / 27$ \\
Age (years) & $11.0 \pm 3.2$ & $8.8 \pm 2.4$ & $8.7 \pm 2.1$ & $12.4 \pm 1.4$ & $9.7 \pm 3.4$ \\
BMI index $\left(\mathrm{kg} / \mathrm{m}^{2}\right)$ & $15.0 \pm 2.6$ & $14.9 \pm 5.8$ & $14.7 \pm 2.4$ & $14.5 \pm 2.5$ & $13.2 \pm 1.6$ \\
FEV $/$ FVC $(\%)$ & $61.0 \pm 15.2^{*+}$ & $59.8 \pm 16.4^{*}$ & $74.0 \pm 18.4^{*}$ & $78.0 \pm 12.4^{*}$ & $85.4 \pm 13.6$ \\
\hline $\mathrm{HDM}-\operatorname{sIgE}\left(\mathrm{KU}_{\mathrm{A}} / \mathrm{L}\right)$ & $76.0 \pm 18.4$ & $69.4 \pm 15.5$ & $71.4 \pm 13.9$ & - & - \\
\hline
\end{tabular}

Data are presented as mean \pm SD.

ASR, asthma combined with allergic rhinitis; AR, allergic rhinitis; AS, bronchial asthma; IR, infective rhinitis; HC, healthy control.

$\mathrm{FEV}_{1}$, forced expiratory volume in 1 second; FVC, forced vital capacity

${ }^{*} P<0.05$, compared with HC group

$+P<0.05$, versus AS, AR or IR.

lymphocytes

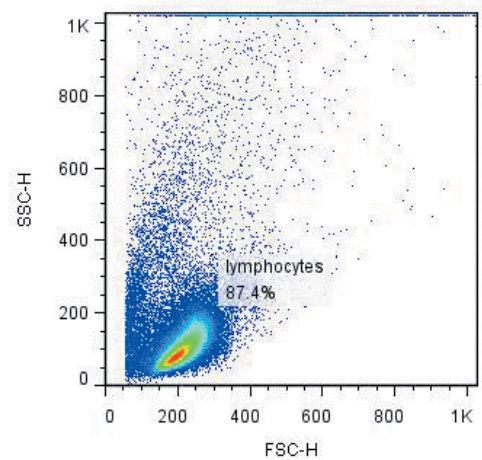

lymphocytes

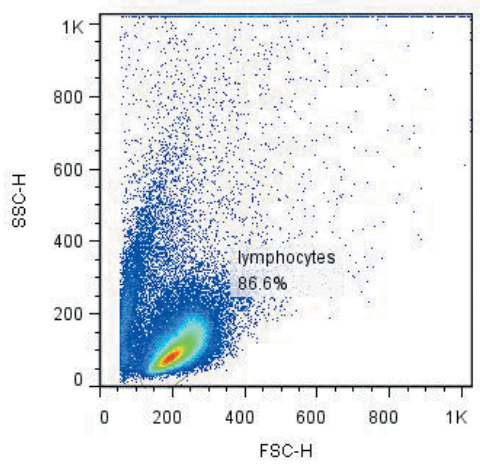

Experimental group

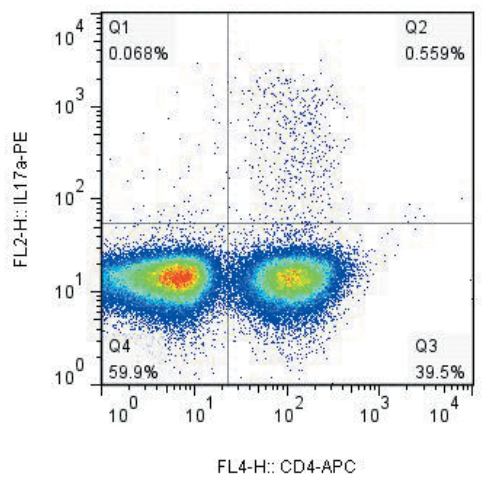

Isotype group

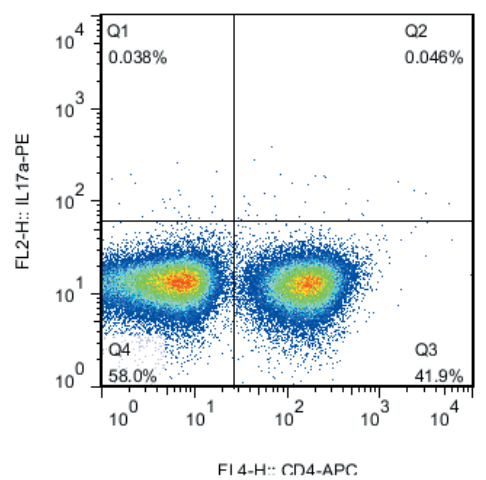

Figure 1. Sequential gating strategy for peripheral blood Th17 cells.

(a) PBMCs were stimulated with PMA and ionomycin for 96 hours; they were then stained with CD4 and the indicated intracellular cytokine (IL-17A). Lymphocytes were gated from PBMCs (left). CD4 IL-17A ${ }^{+}$T lymphocytes were further evaluated by flow cytometry for the intracellular expression of IL-17A or isotype control staining (right). 


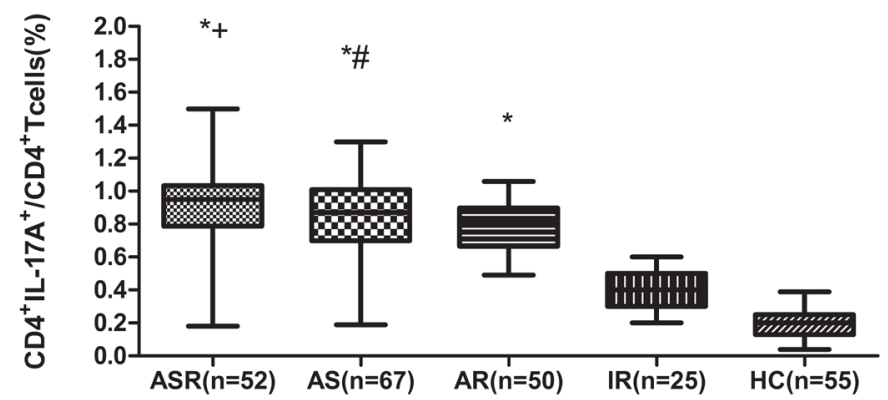

Figure 2. Frequencies of Th17 cell in each group with posive intracellular staining for IL-17A in PBMCS from different groups. Columns represented mean values $\pm \mathrm{SD}$.

ASR, asthma combined with allergic rhinitis; AR, allergic rhinitis; AS, bronchial asthma; IR, infective rhinitis; HC, healthy control.

${ }^{*} P<0.05$, versus healthy control groups.

${ }^{+} P<0.05$, versus AS, AR or IR.

${ }^{\#} P<0.05$, versus AR or IR.

which were higher than those children with infectious rhinitis $[(0.41 \pm 0.15) \%]($ all $P<0.05$, respectively).

\section{Th2-, Th17-Related Cytokines in Plasma or Activated PBMC} Supernatant

Level of Th2-(IL-4, IL-5) and Th17-related (IL-17A, IL $-17 \mathrm{E}$ and IL-17F) cytokines in the plasma or PBMC culture supernatant were measured by ELISA. As shown in Table 2, the median concentrations of IL-4, IL-5, IL-17A, IL-17E, and IL-17F were significantly higher than that in HC group (all $P<0.05$, respectively). A further comparison was performed between patients with allergic disease and infectious rhinitis,

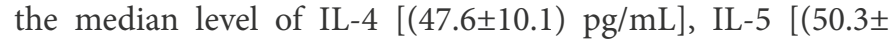
16.1) $\mathrm{pg} / \mathrm{mL}]$ and IL-17E $[(29.8 \pm 6.7) \mathrm{pg} / \mathrm{mL}]$ were obviously

Table 2. Level of Th2 or Th17 Cell Related Cytokines in plasma from each group $(\mathrm{pg} / \mathrm{mL})$

\begin{tabular}{lcccccc}
\hline & $\mathbf{n}$ & IL-4 & IL-5 & IL-17A & IL-17E & IL-17F \\
\hline ASR & 52 & $50.2 \pm 12.5$ & $55.8 \pm 12.3$ & $10.3 \pm 7.8$ & $16.3 \pm 5.2$ & $5.9 \pm 2.6$ \\
\hline AS & 67 & $49.5 \pm 8.9$ & $57.3 \pm 8.1$ & $12.6 \pm 3.8$ & $14.2 \pm 2.5$ & $7.2 \pm 1.2$ \\
\hline AR & 50 & $43.8 \pm 7.8$ & $39.2 \pm 7.2$ & $15.9 \pm 4.8$ & $11.3 \pm 5.4$ & $6.4 \pm 3.4$ \\
\hline IR & 25 & $36.4 \pm 9.8$ & $21.4 \pm 4.5$ & $13.2 \pm 2.2$ & $8.2 \pm 3.7$ & $4.2 \pm 1.6$ \\
\hline HC & 55 & $14.2 \pm 5.6$ & $10.8 \pm 4.8$ & $6.6 \pm 2.5$ & $3.2 \pm 2.4$ & $3.2 \pm 2.3$ \\
\hline$P^{*}$ & - & $<\mathbf{0 . 0 5}$ & $<\mathbf{0 . 0 5}$ & $>0.05$ & $<\mathbf{0 . 0 5}$ & $>0.05$ \\
\hline
\end{tabular}

Data are presented as mean \pm SD.

ASR, asthma combined with allergic rhinitis; AR, allergic rhinitis; AS, bronchial asthma; IR, infective rhinitis; HC, healthy control.

* comparing among five groups

(a) IL-4

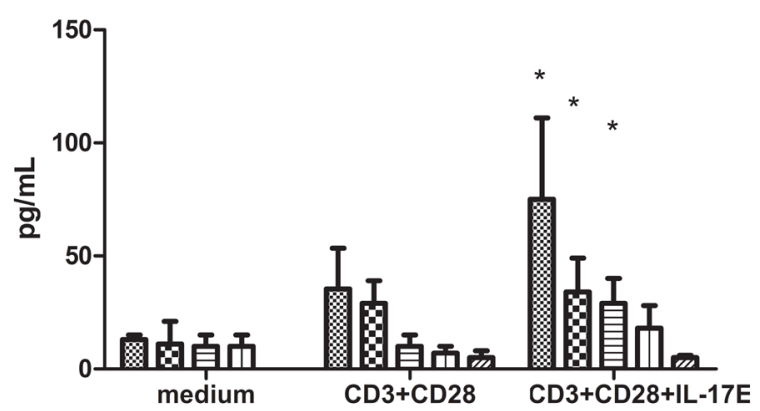

(b) IL-5

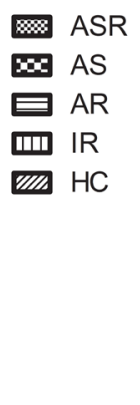

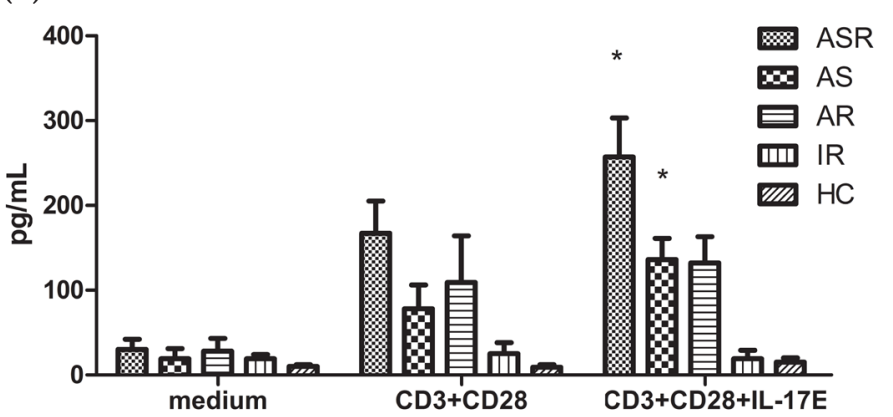

Figure 3. IL-17E-induced products of IL-4, IL-5, and IL-13 from CD4 ${ }^{+}$Th cells in each group.

Upon costimulation of anti-CD3 and anti-CD28 antibodies, all CD4 $4^{+} \mathrm{Th}$ cells $\left(1 \times 10^{6} / \mathrm{mL}\right)$ were cultured in the presence or absence of IL-17E (50 ng/mL) for 96 hours. The release of Th2-related cytokines (IL-4, IL-5 and IL-13) in the supernatant was detected by ELISA. Results are expressed as mean \pm SD from 3 independent experiments.

ASR, asthma combined with allergic rhinitis; AR, allergic rhinitis; AS, bronchial asthma; IR, infective rhinitis; HC, healthy control. ${ }^{*} P<0.05$ when compared with medium control or costimulation conditions (anti-CD3 plus anti-CD28 antibodies) 
(c) IL-13

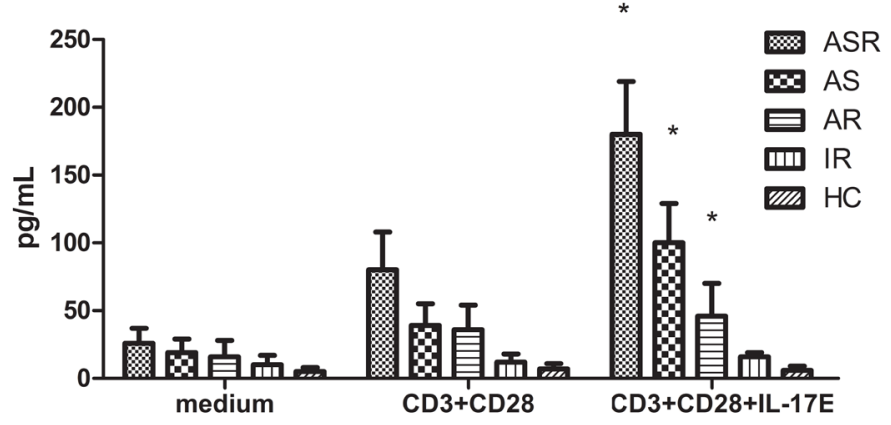

Figure 3. (Continued) IL-17E-induced products of IL-4, IL-5, and IL-13 from $\mathrm{CD4}^{+} \mathrm{Th}$ cells in each group.

higher in children with allergic disease than that in IR group (IL-4 $[(36.4 \pm 9.8) \mathrm{pg} / \mathrm{mL}], \mathrm{IL}-5[(21.4 \pm 4.5) \mathrm{pg} / \mathrm{mL}]$ and IL-17E $[(8.2 \pm 3.7)$ $\mathrm{pg} / \mathrm{mL}]$ ), and the difference were significant (all $P<0.05$, respectively). After stimulation with HDM antigen, it was found that levels of IL-4 and IL-17E in PBMCs culture supernant from allergic children (AS group, AR group and ASR group) were significantly enhanced. However, no obvious changes in Th2 or Th17 cell related cytokines were observed in HDM-activated PBMCs from IR group or HC group (all $P>0.05$, respectively) (Table 3).

\section{Measurement of Th2 Cytokine Production from IL-17E-Activated Th Cells}

In order to explore the biological effect of IL-17E, we next investigated whether there were differences in the production of Th2 cytokines from Th cells with or without IL-17E. As shown in Figure 3, it was revealed that the IL-17E exhibited synergistic effect to induce Th cells from allergic children to product IL-4, IL-5 and IL-13 significantly when comparing with the medium control, or with the costimulation (via anti-CD3/anti-CD28 antibodies). However,it did not show any effect on Th2 type cytokines production from IL-17E treated Th cells in IR group or in HC group (all $P>0.05$, respectively).

\section{Discussion}

Allergic rhinitis (AR) and allergic asthma (AS) are chronic inflammatory diseases in childhood, which are broadly recognized by frequently co-existence in the same individual. Although both AS and AR are considered as manifestations of one syndrome in two parts of the respiratory tract, both sharing some common pathological aspects, it is becoming evident that not all patients with AR would develop asthma eventually in real-world clinical practice. To date, there have been some studies reporting that some differences (such as: genetic susceptibility, local immunologic responses in airway, or environmental allergen exposure) indeed existed among asthmatic patient, allergic rhinitis patients and those with both diseases. ${ }^{15-16}$ Th17 cells are novel $\mathrm{CD}^{+} \mathrm{T}$ subsets with preferential synthesis of IL-17 family cytokines, especially IL-7A and IL-17E, which are thought to play a vital role in the pathogenesis of inflammatory diseases. ${ }^{17-18}$ Previous studies demonstrated that an increase in Th17 cells and related cytokines were observed in local inflammatory sites, such as: bronchial biopsies, bronchoalveolar/ nasal lavage fluid, and sputum samples. ${ }^{19}$ Moreover, genetic evidence also indicated that some polymorphisms occurring in interleukin(IL) -17 gene locus were possibly associated with AR and comorbid asthma in Chinese population. ${ }^{20-21}$ In view of these evidences, we attempted to

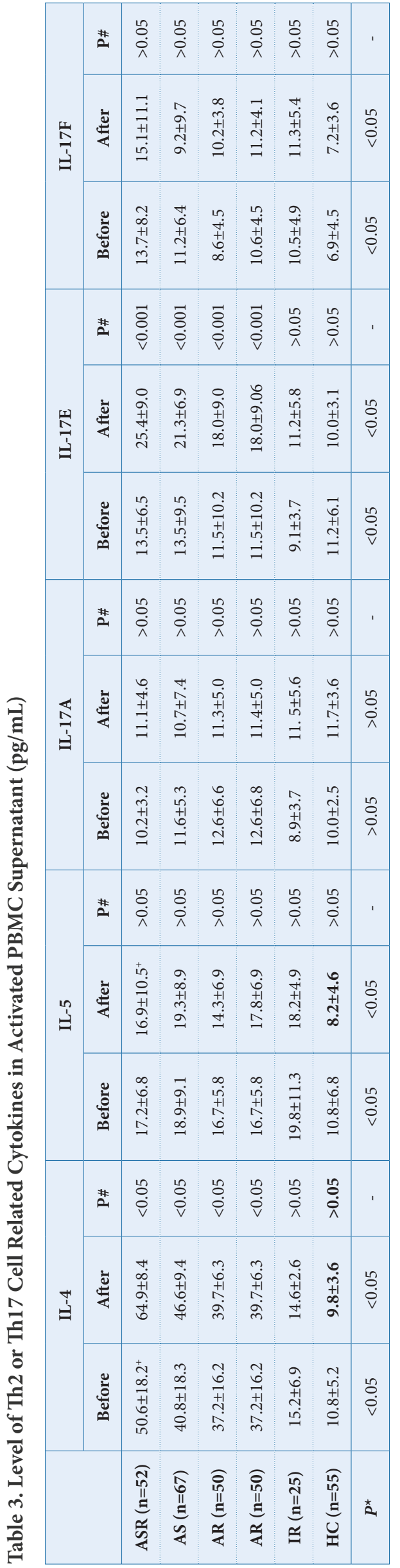


explore whether there were some differences in Th17 cells mediated immunological responses among children with different clinical manifestations of airway inflammation diseases.

According to our results, the percentages of circulating Th17 cells and concentrations of Th2-(IL-4, IL-5 and IL-13), Th17-related cytokines (IL-17A and IL-17E) in plasma were increased in children with allergic diseases when comparing with non-allergic children or with healthy controls. Similar results were reported by Zhao et al, they found the percentages of Th2 and Th17 cells as well as the concentrations of Th2-, Th17-related cytokines were obviously higher in asthmatic that those in healthy subjects. ${ }^{22}$ Our result, as a supplement to the above conclusion, demonstrated that in addition to Th2 pathway, a systematic enhancement of Th17 cell immunity might be involved in immunopathology process of airway allergic diseases. In addition, previous studies on gene expression profile of Th-cell-related genes in asthmatic endobronchial tissue revealed 3 major patient clusters: Th2 high group, Th17 high group, and Th2/Th17-low group. Moreover, comparing with those with Th2 high asthmatics (eosinophils-dominated inflammation), the IL-17 related signaling is more evident in the airway tissue of a subset of patients with moderate-severe asthma. ${ }^{23}$ Although the regulation mechanism in the cellular and molecular processes that govern the interaction between Th2 and Th17 pathway is still unclear, the above findings have emphasized the importance of Th17 immunity in airway inflammation, and its clinical interpretation of this inflammation response still need to be further explored.

Moreover, a obvious enhancement of Th17 cells and IL-17E levels were observed in ASR group than those with AS alone, or AR alone, but the level of HDM-specific $\operatorname{IgE}(\mathrm{sIgE})$ among these three groups was comparable. Another study conducted by Wang et al had also compared the differences in humoral immunity among allergic rhinitis with or without asthma, which showing that a distinctly higher level of sIgE to grass pollen among patient with AR alone than those with ASR. ${ }^{24}$ We assume that the inconsistency is possibly caused by the differences in types of sensitized allergen. There was evidence that within the pollen season a significant increase in clinical symptoms, use of medication and pollen sIgE level were observed among birch-allergic patients. ${ }^{25}$

Finally, we observed that upon IL-17E-mediated activation as well as costimulatory signals (anti-CD3 plus anti-CD28 antibodies), the total Th cells from allergic patients could be induced to release Th2 cytokines in vitro, like: IL-4, IL-5, and IL-13. IL-17E was first identified by Lee et al. in 2001, which was proved as a novel pro-inflammatory cytokine in regulation of eosinophil-dominant airway inflammation. ${ }^{26}$ Intranasal administration of IL-17E has been shown to induce the production of Th2 cytokines and the expression of chemokine eotaxin mRNA in the murine lung, resulting in Th2-like response. ${ }^{27}$ Furthermore, it had been proven that under allergic conditions the expression of IL-17E receptors (IL-17RB) was unregulated on human $\mathrm{CD} 4^{+} \mathrm{Th} 2$ cells, which could be a potential cellular target of IL-17[28]. The study of Wong et al. proved that costimulatory signals can upregulate the expression IL-17E receptors (IL-17RB) on Th cells by activating the JNK and NF- $\kappa B$ pathways, which could further stimulate the response to exogeous IL-17E. ${ }^{29}$ In contrast, it was found in asthmatic mice that the IL-17E-induced enhancement of allergic airway inflammation was inhibited by the depletion of $\mathrm{CD} 4^{+}$Th2 cells. ${ }^{30}$ Therefore, according to the above results, it is indicative that IL-17E may function as an important regulatory cytokine and involve in the development Th2 cell-mediated allergic diseases.

In conclusion, the elevated circulating Th17 cells and IL -17E are important features of airway allergic diseases, which might play a vital role in the regulation of airway inflammation process. A limitation of our study is that it has not fully explored the upstream signals in driving peripheral Th17 cell generation and maturation. Moreover, the cellular cross-talks between Th17 cells and $\mathrm{CD}^{+} \mathrm{T}$ cells population in local microenvironment need to be demonstrated in future. All of these should be based on larger longitudinal populations and will hopefully provide new insights on Th17 immunity in allergic inflammatory diseases of the airways.

\section{Acknowledgements}

This work was supported by a grant from the Beijing Municipal Natural Science Foundation (No. 7172074), and by Beijing Municipal Administration of Hospitals Incubating Program (No. PX2018049).

\section{Conflict of Interest Statement}

We declare that we have no conflict of interest.

\section{References}

1. Lambrecht BN, Hammad H. The immunology of asthma. Nat Immunol. 2015; 16:45-56.

2. Lang K, Allen-Ramey F, Huang H, Rock M, Kaufman E, Dykewicz MS Health care resource use and associated costs among patients with seasonal versus perennial allergic rhinitis. Allergy Asthma Proc. 2016;37:103-11.

3. Tsiakiris G, Neely G, Lind N, Nordin S. Comorbidity in allergic asthma and allergic rhinitis: functional somatic syndromes. Psychol Health Med [Preprint]. 2016 [cited 2016 Dec 30]: [6 p.]. Available from: http://www.tandfonline.com/doi/abs/10.1080/13548506.2016.1276606

4. Asher MI, Weilland SK. The International Study of Asthma and Allergies in Childhood (ISAAC).ISAAC Steering Committee.Clin Exp Allergy.1998; 28:52-66.

5. Strachan D, Sibbald B, Weiland S, Aït-Khaled N, Anabwani G, Anderson $\mathrm{HR}$, et al. Worldwide variations in prevalence of symptoms of allergic rhino conjunctivitis in children: the International Study of Asthma and Allergies in Childhood (ISAAC). Pediatr Allergy Immunol.1997,8:161-76.

6. Giavina-Bianchi P, Aun MV, Takejima P, Kalil J, Agondi RC. United airway disease: current perspectives. J Asthma Allergy. 2016;9:93-100.

7. Amin K. The Role of the T lymphocytes and Remodeling in Asthma Inflammation. Inflammation.2016;39:1475-82

8. Pelaia G, Vatrella A, Busceti MT, Gallelli L, Calabrese C, Terracciano R, et al. Cellular mechanisms underlying eosinophilic and neutrophilic airway inflammation in asthma. Mediators Inflamm. 2015;2015:879783.

9. Park H, Li Z, Yang XO, Chang SH, Nurieva R, Wang YH, et al. A distinct lineage of CD4 T cells regulates tissue inflammation by producing interleukin 17. Nat Immunol. 2005;6:1133-41.

10. Ciprandi G, Filaci G, Fenoglio D. Th17 cells and allergic rhinitis: Is there clinical relevance? Otolaryngol Head Neck Surg. 2010;143:604-5.

11. Shi YH, Shi GC, Wan HY, Jiang LH, Ai XY, Zhu HX, et al. Coexistence of Th1/Th2 and Th17/Treg imbalances in patients with allergic asthma. Chin Med J (Engl). 2011;124:1951-56.

12. Vocca L, Di Sano C, Uasuf CG, Sala A, Riccobono L, Gangemi S, et al. IL-33/ST2 axis controls Th2/IL-31 and Th17 immune response in allergic airway diseases. Immunobiology. 2015;220:954-63.

13. Global Initiative for Asthma(GINA).Global strategy for asthma mangment and preventition.2015(revision)[EB/OL].URL: http://www.ginaasthma.org 
14. Bousquet J, Schünemann HJ, Samolinski B, Demoly P, Baena-Cagnani CE, Bachert C, et al. Allergic Rhinitis and its Impact on Asthma (ARIA): achievements in 10 years and future needs.J Allergy Clin Immunol. 2012;130:1049-62.

15. Dizier MH, Bouzigon E, Guilloud-Bataille M, Genin E, Oryszczyn MP, Annesi-Maesano I, et al. Evidence for a locus in 1p31 region specifically linked to the co-morbidity of asthma and allergic rhinitis in the EGEA study. Hum Hered. 2007;63:162-7.

16. Dogru M. Investigation of asthma comorbidity in children with different severities of allergic rhinitis. Am J Rhinol Allergy. 2016;30:186-9.

17. Yao X, Sun Y, Wang W, Sun Y. Interleukin (IL)-25: Pleiotropic roles in asthma. Respirology. 2016;21:638-47.

18. Pene J, Chevalier S, Preisser L, Venereau E, Guilleux MH, Ghannam S, et al. Chronically inflamed human tissues are infiltrated by highly differentiated Th17 lymphocytes. J Immunol. 2008;180:7423-30.

19. Bullens DM, Truyen E, Coteur L, Dilissen E, Hellings PW, Dupont LJ, et al. IL-17 mRNA in sputum of asthmatic patients: linking T cell driven inflammation and granulocytic influx? Respir Res. 2006;7:1-9.

20. Wang M, Zhang Y, Han D, Zhang L. Association between polymorphisms in cytokine genes IL-17A and IL-17F and development of allergic rhinitis and comorbid asthma in Chinese subjects. Hum Immunol. 2012;73:647-53.

21. Fogli LK, Sundrud MS, Goel S, Bajwa S, Jensen K, Derudder E, et al. T cell-derived IL-17 mediates epithelial changes in the airway and drives pulmonary neutrophilia. J Immunol. 2013;191:3100-11.

22. Zhao Y, Yang J, Gao YD, Guo W. Th17 immunity in patients with allergic asthma. Int Arch Allergy Immunol. 2010;151:297-307.
23. Choy DF, Hart KM, Borthwick LA, Shikotra A, Nagarkar DR, Siddiqui S, et al. TH2 and TH17 inflammatory pathways are reciprocally regulated in asthma. Sci Transl Med. 2015 19;7:301ra129.

24. Wang WY, Boot JD, Mascelli MA, Gerth van Wijk R, Diamant Z. Comparison of biomarkers between allergic rhinitis only and allergic rhinitis with concomitant asthma. Allergy. 2009;64:1102-3.

25. Wosinska-Becler K, Plewako H, Håkansson L, Rak S. Cytokine production in peripheral blood cells during and outside the pollen season in birch -allergic patients and non-allergic controls. Clin Exp Allergy. 2004;34: 123-30.

26. Lee J, Ho WH, Maruoka M, Corpuz RT, Baldwin DT, Foster JS, et al. IL-17E, a novel proinflammatory ligand for the IL-17 receptor homolog IL-17Rh1. J Biol Chem. 2001;276:1660-4.

27. Fort MM, Cheung J, Yen D, Li J, Zurawski SM, Lo S, et al. IL-25 induces IL-4, IL-5, and IL-13 and Th2-associated pathologies in vivo. Immunity. 2001;15:985-95.

28. Wang YH, Angkasekwinai P, Lu N, Voo KS, Arima K, Hanabuchi S, et al. IL-25 augments type 2 immune responses by enhancing the expansion and functions of TSLP-DC-activated Th2 memory cells. J Exp Med. 2007,6; 204:1837-47.

29. Wong CK, Li PW, Lam CW. Intracellular JNK, p38 MAPK and NF-kappaB regulate IL-25 induced release of cytokines and chemokines from costimulated T helper lymphocytes. Immunol Lett. 2007;112:82-91.

30. Tamachi T, Maezawa Y, Ikeda K, Kagami S, Hatano M, Seto Y, et al. IL-25 enhances allergic airway inflammation by amplifying a TH2 cell-dependent pathway in mice. J Allergy Clin Immunol. 2006;118:606-14. 